Classification

Physics Abstracts

$10.54-11.10$

\title{
MESURE DE LA MULTIPLICITÉ DES NEUTRONS DE FISSION AÚ MOYEN D'UN SCINTILLATEUR DE GRAND VOLUME CHARGÉ AU GADOLINIUM
}

\author{
M. RIBRAG, J. POITOU, J. MATUSZEK et C. SIGNARBIEUX \\ Service de la Métrologie et de la Physique Neutroniques Fondamentales \\ 'Centre d'Etudes Nucléaires de Saclay, 91-Gif-sur-Yvette, France
}

(Reçu le 17 décembre 1971, révisé le 7 mars 1972)

\begin{abstract}
Résumé. - Un scintillateur liquide de grand volume, chargé au gadolinium, est utilisé à Saclay pour mesurer la multiplicité des neutrons émis dans la fission ; nous décrivons en détail une procédure originale de mise au point de l'ensemble (détecteur-électronique associée) destinée à minimiser les risques d'erreur systématique. La résolution du problème des diverses corrections et le calcul des erreurs associées sont présentés explicitement. Des résultats concernant la fission spontanée de ${ }^{25}{ }^{2} \mathrm{Cf}$ sont comparés aux données de la littérature.

Abstract. - A large liquid scintillator filled with gadolinium and used at Saclay for fission neutron-multiplicity experiments, is described. An original procedure for optimal adjustments of the detecting system and its associated electronics so as to minimize possible systematic errors is also presented. Several errors and their associated corrections are treated in detail. Results for spontaneous fission of ${ }^{252} \mathrm{Cf}$ are compared with the data published by others.
\end{abstract}

Introduction. - Pour mesurer la multiplicité des neutrons émis dans une réaction nucléaire, un scintillateur liquide de grand volume et chargé au gadolinium, paraît être le détecteur idéal : son efficacité intrinsèque est pratiquement indépendante de l'énergie des neutrons incidents [1] et peut avoir une valeur élevée $(\sim 90 \%)$ tout en conservant un excellent rapport signal sur bruit. Ce type de détecteur a donc été fréquemment utilisé, notamment pour des mesures de sections efficaces photonucléaires $(\gamma, x n)$ [2], [4] et pour l'étude de l'émission neutronique dans la fission des noyaux lourds [5], [10]; la valeur absolue de l'efficacité de détection est alors toujours déterminée par référence à la valeur absolue du nombre moyen $\bar{v}$ de neutrons prompts émis dans la fission spontanée de ${ }^{252} \mathrm{Cf}$. Or, il existe depuis longtemps un désaccord systématique (de l'ordre de $3 \%$ ) entre les mesures absolues de $\bar{v}$ réalisées par la technique du scintillateur liquide et celles obtenues par d'autres méthodes [11]. Ce fait nous a incités à reprendre en détail le problème des erreurs systématiques dans la technique du scintillateur liquide, à l'occassion de la mise en œuvre à Saclay d'un tel détecteur destiné à l'étude approfondie de l'émission neutronique par les fragments de fission.

Notre détecteur est un scintillateur liquide de 500 litres, composé essentiellement de xylène et chargé en gadolinium à la concentration de $0,5 \%$ en poids. Rappelons brièvement le processus de détection par un tel détecteur de $n$ neutrons émis simultanément :

- Les $n$ neutrons sont ralentis quasi-instantané- ment par chocs nucléaires successifs sur les noyaux d'hydrogène du xylène : les protons de recul et les rayons gamma prompts de la réaction nucléaire créent dans le scintillateur un signal en phase avec l'événement nucléaire.

- Les $n$ neutrons thermalisés diffusent dans le scintillateur avec une vie moyenne d'environ $10 \mu \mathrm{s}$ avant d'être capturés à des instants différents par les noyaux de gadolinium.

- Chaque neutron est détecté individuellement par scintillation sous l'effet des gammas de capture du gadolinium.

Expérimentalement le problème se pose en ces termes :

- Il faut compter les neutrons dans un intervalle de temps judicieusement choisi ; la porte de comptage sera déclenchée par un signal de détection auxiliaire de l'événement nucléaire avec un retard suffisant pour exclure sans ambiguitté le signal prompt du scintillateur dû aux gammas prompts et aux protons de recul.

- Il faut mesurer le bruit de fond ambiant dans une porte de comptage de largeur identique et non corrélée aux événements nucléaires étudiés.

- Il faut éliminer avec soin les causes d'erreurs systématiques dont la principale consiste à compter plusieurs coups pour un même neutron.

- Il faut connaître avéc précision le temps mort du dispositif de comptage car les corrections deviennent rapidement importantes avec le degré de multi- 
plicité des neutrons. Ces corrections de temps mort impliquent également la connaissance de la distribution de probabilité des temps de capture dans le scintillateur.

Après avoir justifié et décrit le choix des matériels utilisés, nous nous proposons de décrire en détail une procédure de réglage et d'association des tubes photomultiplicateurs, procédure susceptible d'éliminer les causes d'erreurs systématiques. Nous présentons ensuite un certain nombre de courbes qui caractérisent les paramètres les plus utiles de l'ensemble instrumental (temps de coïncidence, temps mort, distribution des temps de capture, rapport signal/ bruit). Nous développons, d'autre part, les méthodes de calcul pour effectuer les corrections sur les données brutes et évaluer les erreurs; enfin, nous proposons un critère original destiné à tester sans ambiguïté la qualité de l'ensemble de la mesure.

1. Description du détecteur - Notre détecteur est constitué d'une enveloppe sphérique de $0,50 \mathrm{~m}$ de rayon revêtue intérieurement d'une couche de peinture diffusante à l'oxyde de titane; il est muni d'un tube axial de $10 \mathrm{~cm}$ de diamètre permettant d'installer, en son centre, la cible et un dispositif de mesure complémentaire. Il est rempli de liquide scintillant NE 323 dont les caractéristiques essentielles sont les suivantes : indice de réfraction : 1,5; constante de temps de décroissance lumineuse : $3,8 \mathrm{~ns}$; maximum du spectre de fréquence de la lumière émise : $4250 \AA$. Le scintillateur est vu par 8 photomultiplicateurs. Pour un scintillateur de dimension aussi importante, la somme des surfaces des photocathodes des tubes est très faible par rapport à la surface de l'enveloppe; il s'ensuit que l'angle solide de vision directe de la lumière est très petit (inférieur à $1 \%$ ) et que la collection lumineuse n'est totale qu'après de nombreuses diffusions de la lumière sur les parois. Dans ces conditions, l'impulsion lumineuse ne sera pas très brève et il n'est donc pas indispensable de choisir des tubes avec des temps de montée rapides. Nous avons utilisé des tubes Radiotechnique 54 DVP pour les raisons suivantes : i) grand diamètre de la photocathode : $110 \mathrm{~mm}$; ii) rendement quantique de la photocathode de type « $\mathrm{D}$ » supérieur de $50 \%$ à celui des cathodes de type « S $11 »$ pour $\lambda=4250 \AA$; iii) meilleur piégeage des ions $C s$ dans ce type de cathode, ce qui conduit à une meilleure stabilité du bruit dans le temps (absence de migration des ions Cs vers les dynodes).

2. Réglage et association des photomultiplicateurs. - Le problème à résoudre est d'additionner en phase les impulsions d'anode des différents P. M.

Ceci implique pour chaque P. M. d'une part, de minimiser la dispersion des temps de transit due à l'écart centre-bord de la photocathode et d'autre part, d'éviter, autant que faire se peut, la création d'impulsions satellites («post-impulsions »). Ce phénomène parasite existe en effet quels que soient les tubes utilisés ; le nombre et l'amplitude de ces impulsions sont en moyenne d'autant plus grands que la quantité de lumière reçue par la photocathode est grande et la tension d'accélération du tube élevée. Par examen d'oscillogrammes, il est possible d'en compter plusieurs par microseconde, dans les premières microsecondes qui suivent l'impulsion initiale. Il devient alors évident qu'il est très difficile de s'affranchir totalement de ce grave inconvénient, même au moyen de coïncidences rapides entre deux groupes de tubes. Une solution aisée consiste à diminuer la haute tension appliquée sur le tube ; on peut pratiquement éliminer les post-impulsions avec une tension de polarisation de 1000 à $1100 \mathrm{~V}$. Cette solution est doublement avantageuse : i) diminution considérable du bruit de fond électronique du tube et de son vieillissement; ii) possibilité d'appliquer la H. T. sur la photocathode (ce qui serait certainement à proscrire avec une tension élevée pour éviter une instabilité du bruit cathodique) : le montage " anode à la masse " permet de supprimer la capacité d'isolement qui introduit une constante de temps de différenciation, donc un temps mort variable dépendant de l'amplitude de l'impulsion.

L'association des P. M. nécessite au préalable l'égalisation de leurs gains. La disposition géométrique des huit tubes étant identique par rapport au centre du détecteur, cette opération consiste à ajuster la tension de polarisation de chaque tube de façon à rendre égales leurs efficacités de détection aux neutrons de fission émis par une source de ${ }^{252} \mathrm{Cf}$ placée au centre du détecteur. Nous avons envisagé deux types de groupement des P. M. : montage « SOMME » (Fig. 1A) dans lequel il est fait la somme analogique des signaux de toutes les voies pour attaquer un discriminateur d'amplitude; montage " COÏNCIDENCE » (Fig. 1B) dans lequel il est fait une coïncidence rapide entre deux voies qui résultent chacune de l'addition de 4 P. M.

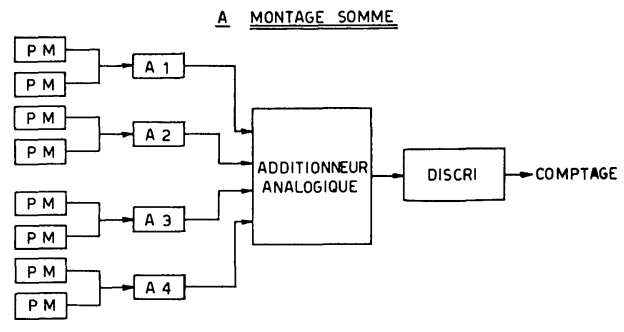

B MONTAGE COINCIDENCE

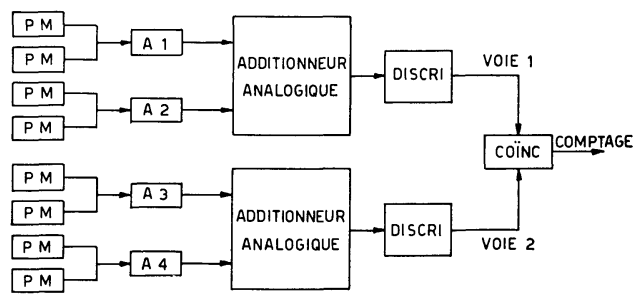

FIG. 1. - Dispositifs de groupement des tubes. 
a) Montage « SOMME ». - Etude de la réponse en amplitude du détecteur.

Pour effectuer cette mesure, nous avons remplacé le discriminateur de la figure $1 \mathrm{~A}$ par un amplificateur lent (ORTEC 450) et nous avons analysé la distribution des amplitudes des impulsions.

La figure 2 représente : i) le bruit de fond nucléaire

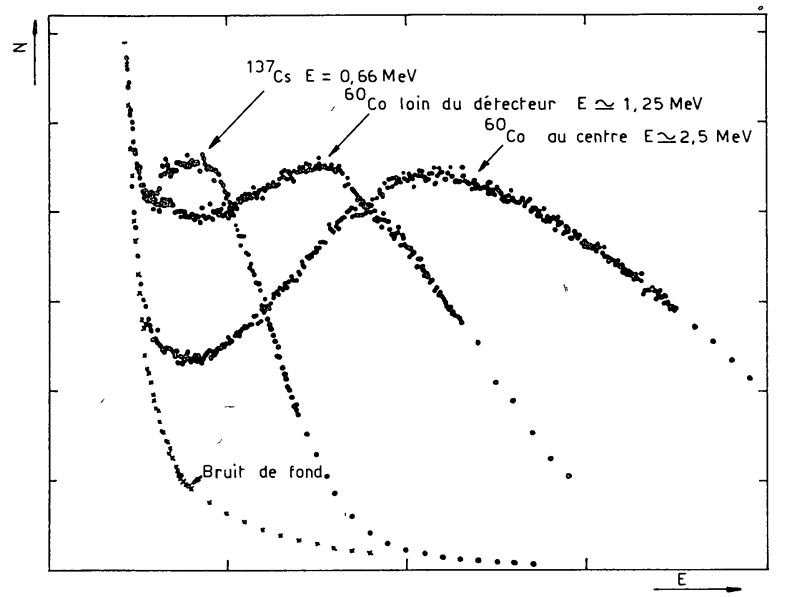

FIG. 2. - Réponse en amplitude du détecteur aux rayonnements $\gamma$ de ${ }^{137} \mathrm{Cs}$ et ${ }^{60} \mathrm{Co}$ et du bruit de fond ambiant dans les mêmes conditions.

ambiant; ii) le spectre obtenu en plaçant au centre du détecteur une source de ${ }^{137} \mathrm{Cs}: E=660 \mathrm{keV}$; iii) le spectre obtenu en plaçant une source de ${ }^{60} \mathrm{Co}$ loin du détecteur, on peut admettre que, dans ce cas, l'énergie moyenne est de l'ordre de $1,25 \mathrm{MeV}$; iv) le spectre obtenu en plaçant la même source de ${ }^{60} \mathrm{Co}$ au centre du détecteur : l'énergie moyenne est alors de $2,5 \mathrm{MeV}$.

La figure 3 représente: i) la distribution des

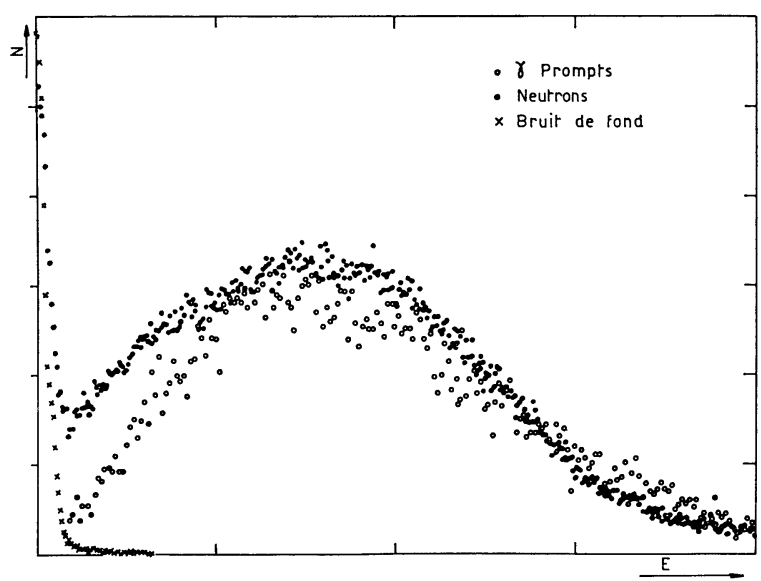

FIG. 3. - Distributions des amplitudes des $\gamma$ prompts de fission, des $\gamma$ de capture des neútrons de fission et du bruit de fond dans les mêmes conditions.

amplitudes des rayonnements gamma de fission ; ii) la distribution des amplitudes des rayonnements gamma consécutifs à la capture d'un neutron par le gadoli- nium; iii) la distribution des amplitudes du bruit nucléaire ambiant. Il est à remarquer que le spectre de gamma de fission est mieux résolu que le spectre de gamma de capture. Ceci s'explique par le fait que les rayons gamma de fission sont émis au centre du détecteur et que leur énergie moyenne est faible ; par conséquent, les interactions avec le scintillateur ont lieu au voisinage du centre du détecteur. Au contraire, les neutrons ont une probabilité appréciable d'être capturés près de la surface du détecteur, ce qui peut produire un "échappement" important des rayons gamma de capture.

b) Montage «COÏNCIDENCE ». - Etude de la réponse en temps du détecteur.

La figure 4 montre la distribution des écarts de

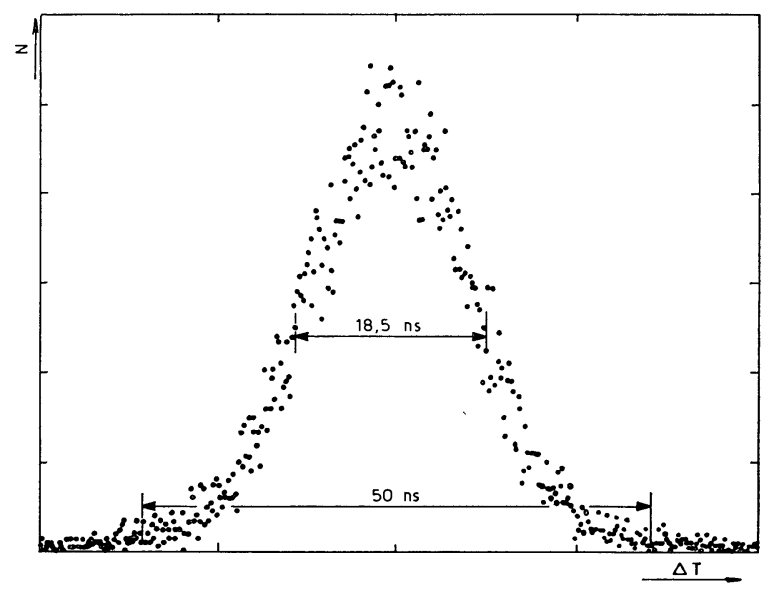

FIG. 4. - Mesure du temps de coïncidence. Distribution des différences de temps de passage au seuil pour deux groupes de quatre tubes.

temps entre les impulsions provenant de deux groupes de tubes. Un temps de coïncidence $2 \tau=50$ ns correspond à la détection de plus de $99 \%$ des coups.

3. Mesure du temps mort et du rapport signal/bruit. - Nous avons comparé les mérites des deux montages «SOMME » et «COÏNCIDENCE » relativement aux deux paramètres qui nécessitent d'apporter des corrections aux données mesurées: le temps mort et le rapport signal/bruit.

3.1 MESURE DU TEMPS MORT INTRINSÈQUE. - La méthode consiste à mesurer la distribution de probabilité du temps qui sépare deux impulsions - neutron (sélectionnées par la porte de comptage). Expérimentalement, cette distribution est obtenue de la façon suivante : les impulsions de sortie du discriminateur (montage "SOMME» Fig. 1A) ou du circuit de coïncidence (montage " COÏNCIDENCE », Fig. 1B) attaquent, avec un retard fixe $t$, l'entrée " start " d'un CTA et, sans retard, l'entrée "stop » du même CTA. Soient deux impulsions séparées par un intervalle de temps $T$; le CTA analyse l'intervalle de temps $(T-t)$, comme on le voit clairement sur la figure 5. 


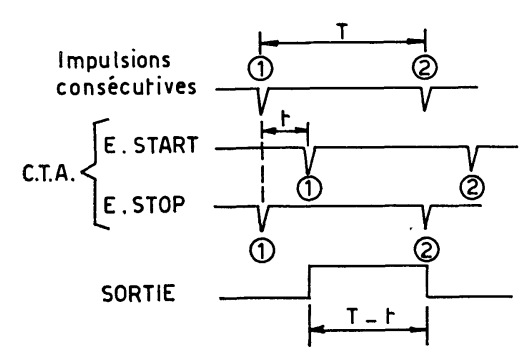

Fig. 5. - Schéma logique de la méthode de mesure des temps morts.

Sur la figure 6 sont représentées les distributions mesurées par cette méthode avec le montage « SOMME » pour des seuils de discrimination corres-

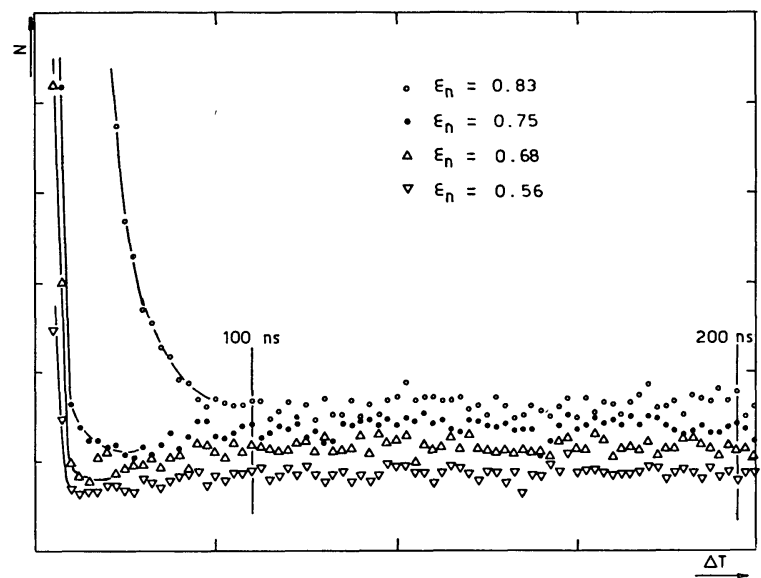

FIG. 6. - Mesure du temps mort : montage "SOMME». Distribution des écarts entre les temps d'arrivée de deux impulsions consécutives pour différentes efficacités de détection neutronique.

pondant à des efficacités aux neutrons $\varepsilon_{n}$ comprises entre $83 \%$ et $56 \%$ : on constate l'apparition de rebasculements au-dessous d'un temps qui correspond à la largeur de l'impulsion la plus grande, au niveau du seuil de discrimination.

La figure 7 montre la même distribution pour le montage «COÏNCIDENCE », l'efficacité de détec-

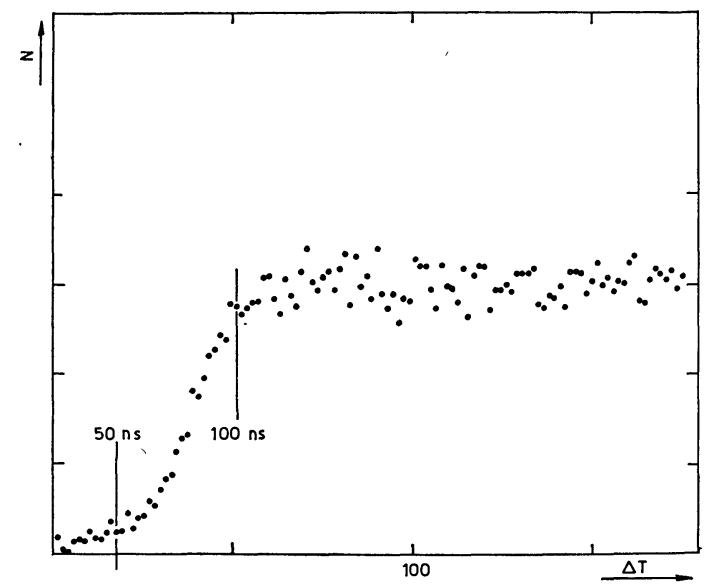

Fig. 7. - Mesure du temps mort : montage $\ll$ COÏNCIDENCE ». tion des neutrons étant égale à $82 \%$. On peut noter que les coïncidences rapides ( $2 \tau=50 \mathrm{~ns})$ éliminent les rebasculements; d'autre part, les discriminateurs placés sur les deux voies en coïncidence ont chacun un temps mort minimal de $25 \mathrm{~ns}$ qui correspond à la largeur du signal de sortie : le temps mort sur chaque voie varie donc entre 25 ns et la largeur au seuil de discrimination de l'impulsion la plus grande : ce temps mort intrinsèque explique les pertes de comptage dans la première partie de la distribution montrée sur la figure 7 .

On peut constater que les deux montages « SOMME » et «COÏNCIDENCE » sont équivalents du point de vue du temps mort qui n'est jamais supérieur à 100 ns. Pour la simplicité des corrections, nous avons imposé au système dé comptage un temps mort fixe de 100 ns.

Les deux montages ont également été étudiés en vue de déterminer le rapport signal sur bruit. La séquence électronique d'acquisition des données se compose d'une porte de comptage des neutrons de $35 \mu \mathrm{s}$, retardée de $1 \mu \mathrm{s}$ par rapport à la fission, et, $100 \mu$ s plus tard, d'une porte de comptage du bruit de fond de $35 \mu$ s.

Si $M$ et $B$ sont respectivement les nombres moyens de coups dans la porte-neutron et dans la portebruit et si $\bar{v}$ est le nombre moyen de neutrons émis dans la fission de ${ }^{252} \mathrm{Cf}$, on définit l'efficacité neutronique par :

$$
\varepsilon_{n}=\frac{M-B}{\bar{v}} .
$$

La figure 8 montre une série de courbes obtenues

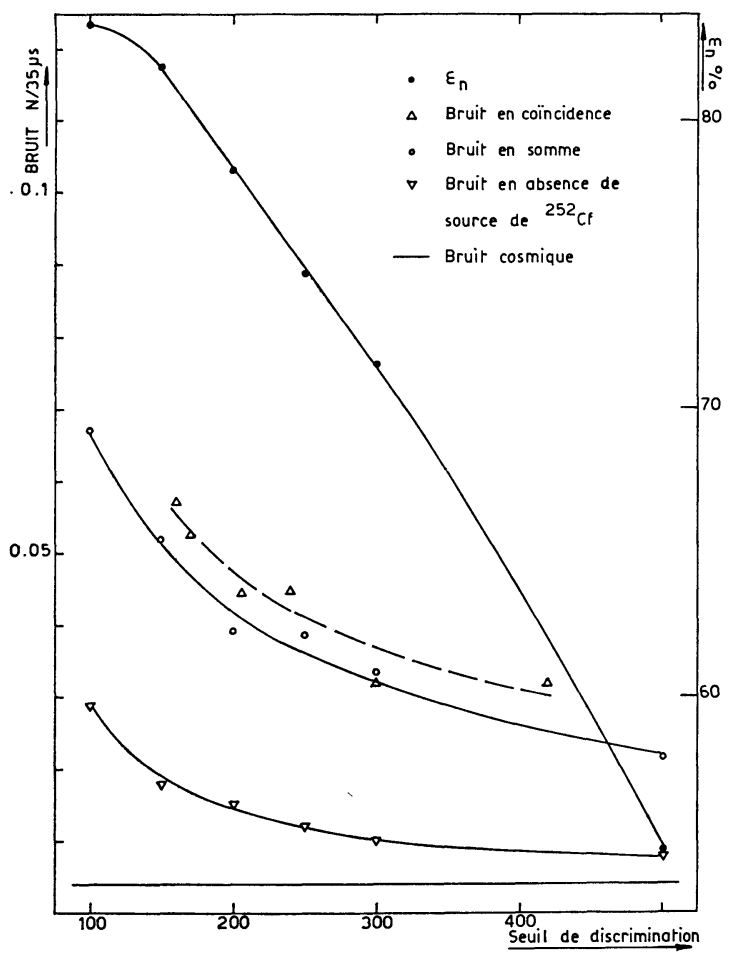

FIG. 8. - Mesures de l'efficacité neutronique et du bruit de fond ambiant en fonction du seuil de détection. 
en fonction $\mathrm{du}$ seuil de détection (la largeur des portes de comptage étant fixée à $35 \mu \mathrm{s}$ ). Pour une efficacité $\varepsilon_{n}$ donnée, on peut remarquer que le bruit de fond en coïncidence est comparable au bruit de fond obtenu lorsque tous les signaux des tubes sont additionnés, ce qui prouve que le bruit détecté est uniquement d'origine nucléaire et que, par conséquent, le montage «COÏNCIDENCE » ne permet pas de le réduire.

Les deux montáges étant strictement équivalents, nous avons donc pour des raisons de simplicité choisi le montage "SOMME ».

4. Détermination de la distribution des temps de capture des neutrons. - La correction de temps mort à appliquer sur la distribution du nombre de neutrons détectés nécessite de connaître : i) la valeur du temps mort fixe adoptée dans l'expérience (voir paragraphe précédent); ii) la distribution des temps de capture des neutrons par les noyaux de gadolinium.

Pour effectuer cette mesure, on doit disposer : i) d'un signal en phase avec l'émission des neutrons : ce sera, dans notre cas, le signal délivré par un détecteur de fragments de fission; ii) du signal de capture d'un neutron avec la condition évidente qu'il n'y ait aucun autre neutron détecté (il s'agit en effet de mesurer le temps de capture d'un neutron unique dans la porte de comptage). Si l'on admet que l'amplitude du signal neutron est indépendante du nombre de neutrons émis (hypothèse raisonnable puisque l'énergie dissipée dans le scintillateur par les rayons gamma de capture est constante et que les variations d'amplitude du signal détecté ne sont dues qu'à la statistique de la collection lumineuse), la façon la plus simple d'opérer est de sélectionner le neutron par l'amplitude du signal détecté, par exemple les amplitudes les plus grandes. Par augmentation du seuil de discrimination, nous avons réduit l'efficacité de détection à $1,3 \%$, ce qui correspond à la détection d'un neutron pour 20 fissions.

La figure 9 représente la distribution des temps de

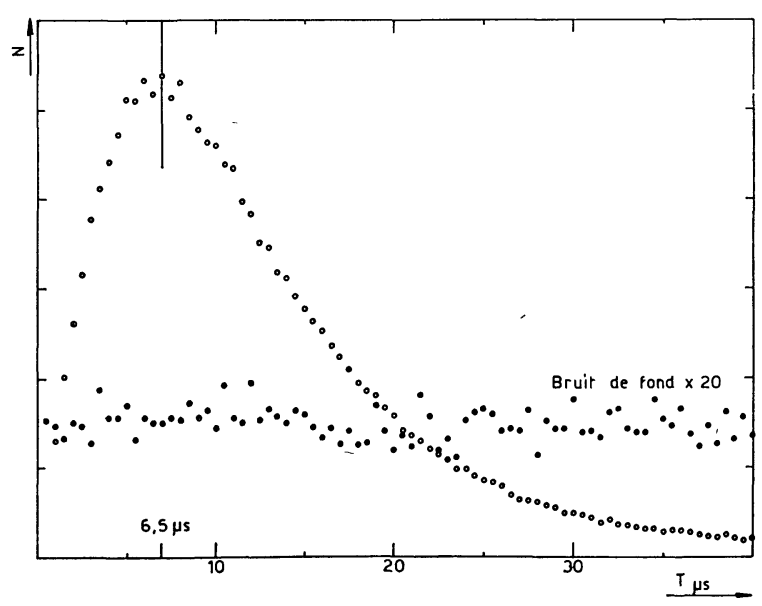

FIG. 9. - Distribution des temps de capture des neutrons dans le scintillateur. capture obtenue jusqu'à $40 \mu$ s après l'émission des neutrons et, dans le même temps d'expérience, la distribution des temps de détection de bruit de fond, les ordonnées étant multipliées par 20. Par soustraction des deux courbes, on obtient la distribution des temps de capture des neutrons nécessaire pour effectuer les corrections de temps mort.

5. Correction des données expérimentales. - Les données de l'expérience sont les suivantes :

$T:$ largeur en temps des deux portes de comptage signal et bruit ;

$\tau$ : temps mort fixe imposé au système de comptage ; notons que ce système est du type "non paralysable » c'est-à-dire qu'une impulsion ayant lieu pendant le temps mort de la précédente, n'introduit pas de temps mort supplémentaire ;

$\mathcal{N}$ : nombre d'événements nucléaires détectés;

$M_{n}$ : distribution du nombre de coups dans la porte-signal (neutron + bruit) ;

$B_{n}$ : distribution du nombre de coups dans la portebruit ;

$\varepsilon$ : efficacité aux neutrons du détecteur.

Pour obtenir la distribution de probabilité $P_{n}$ du nombre de neutrons émis, trois corrections sont à effectuer : i) correction de bruit de fond qui transforme $M_{n}$ en $T_{n}$; ii) correction de temps mort qui transforme $T_{n}$ en $N_{n}$; iii) correction d'efficacité qui transforme $N_{n}$ en $Q_{n}$. Nous obtenons alors :

$$
P_{n}=\frac{Q_{n}}{\sum_{n} Q_{n}}
$$

5.1 CORRECTION DE BRUIT DE FOND. - La distribution de probabilité du bruit de fond $\mathcal{B}_{n}=B_{n} / \Sigma_{n} B_{n}$ est réputée la même dans la porte de comptage du signal et dans la porte de comptage du bruit. Nous adoptons le point de vue que tout recouvrement neutron-bruit sera compté comme un neutron, ce qui revient à le considérer comme une perte de comptage d'un coup de bruit ; on négligera par ailleurs les recouvrements entre un neutron et plusieurs coups de bruit. Dans ces conditions, dans la porte de comptage du signal, la distribution de probabilité $b_{n}^{i}$ du bruit sera, en présence de $i$ neutrons :

$$
b_{n}^{i}=\mathscr{B}_{n}\left(\frac{1-n i \tau}{T}\right)+\frac{\mathfrak{B}_{n+1}(n+1)-i \tau}{T} .
$$

Le système de correction de bruit de fond s'écrit :

$$
M_{n}=\sum_{i=0}^{n} T_{i} b_{n-i}^{i} .
$$

Ce système se résout aisément par récurrence mais, du fait des erreurs statistiques sur $M_{n}$ et $B_{n}$, on obtient en général pour les $n$ élevés (lorsque la probabilité $T_{n}$ devient très faible) des valeurs $T_{n}$ alternativement négatives et positives : nous avons adopté la conven- 
tion d'annuler $T_{n}$ à partir du premier $n$ donnant une probabilité négative ( $n=9$ pour la fission spontanée de ${ }^{252} \mathrm{Cf}$ ).

5.2 CORRECTION DE TEMPS MORT. - La distribution des temps de capture des neutrons montre que, dans le cas de la fission spontanée de ${ }^{252} \mathrm{Cf}$, où il peut y avoir 8 neutrons détectés dans la porte de comptage du signal, le taux de comptage instantané peut atteindre des valeurs de $10^{6}$ coups/s, ce qui implique de faire des corrections de recouvrement d'ordre élevé. Le calcul formel des probabilités de recouvrement de tous ordres est inextricable, nous avons donc déterminé ces probabilités en simulant par la méthode de Monte-Carlo, sur des séries de 10000 événements, la distribution mesurée des temps de capture (Fig. 9); l'existence d'un temps mort fixe de 100 ns se traduit par une matrice de probabilité $\mathcal{C}_{i}^{j}$ de compter effectivement $i$ neutrons quand $j$ sont présents; il s'ensuit que :

$$
T_{i}=\sum_{j=0}^{i} \mathcal{G}_{i}^{j} N_{j}
$$

La matrice $\mathcal{C}_{i}^{j}$ est inversée numériquement à l'aide de l'ordinateur.

5.3 CORRECTION D'efFicACITÉ. - L'efficacité neutronique du détecteur eśt définie par :

$$
\varepsilon=\frac{\sum_{i} i N_{i}}{\bar{v} \times \sum_{i} N_{i}}
$$

$\bar{v}$ étant la valeur absolue du nombre moyen de neutrons émis dans la fission spontanée de ${ }^{252} \mathrm{Cf}$ [11].

Le système de correction d'efficacité s'écrit :

$$
N_{i}=\sum_{j=i}^{\infty} C_{j}^{i} \varepsilon^{i}(1-\varepsilon)^{j-i} Q_{j}
$$

Ce système s'inverse simplement [12] en changeant $\varepsilon$ en $\varepsilon^{-1}$

$$
Q_{j}=\sum_{i=j}^{\infty} C_{i}^{j} \varepsilon^{-j}\left(1-\varepsilon^{-1}\right)^{i-j} N_{i}
$$

La distribution de probabilité du nombre de neutrons émis $\boldsymbol{P}_{\boldsymbol{n}}$ est alors :

$$
P_{n}=\frac{Q_{n}}{\sum_{n} Q_{n}}
$$

5.4 CAlCul Des ERREURS STATISTIQUES. - Le calcul rigoureux des erreurs statistiques sur les $P_{n}$ s'avère inextricable du fait que les matrices de correction sont des fonctions compliquées des paramètres indépendants $M_{n}$ et $B_{n}$; nous avons utilisé une méthode d'évaluation qui consiste à découper l'ensemble de la mesure en $p$ parties égales et à déterminer, pour chaque valeur de $n$, la variance de la distribution des $p$ valeurs de $P_{n}^{p}$. Si les erreurs sont d'origine purement statistique, l'erreur donnée par la relation :

$$
\operatorname{Er}^{2}\left(P_{n}\right)=\frac{\sigma^{2}\left(P_{n}^{n}\right)}{p}
$$

sera indépendante de $p$. La dispersion des résultats en fonction de $p$ est suffisamment faible pour considérer que les erreurs ainsi calculées sont bien représentatives. Notons que, à ces erreurs statistiques, il faudrait ajouter l'erreur liée à l'incertitude sur la valeur absolue de $\bar{v}$ de ${ }^{252} \mathrm{Cf}$, valeur de référence pour le calcul de $\varepsilon$.

6. Critère pour tester la qualité de la mesure. On peut caractériser la distribution $P_{n}$ des neutrons par un paramètre $R$, dépendant de sa forme, invariant par rapport à l'efficacité de détection des neutrons. En effet, les valeurs moyennes $\bar{N}$ et $\bar{n}$ et les variances $\sigma_{N}^{2}$ et $\sigma_{n}^{2}$ des distributions $N_{n}$ et $P_{n}$ avant et après correction de l'efficacité $\varepsilon$, sont reliées [12] par la relation :

$$
\sigma_{n}^{2}=\varepsilon^{-2} \sigma_{N}^{2}+\varepsilon^{-1}\left(1-\varepsilon^{-1}\right) \bar{N},
$$

avec :

$$
\varepsilon=\frac{\bar{N}}{\bar{n}}
$$

on a donc:

$$
\frac{\sigma_{n}^{2}}{\bar{n}^{2}}-\frac{1}{\bar{n}}=\frac{\sigma_{N}^{2}}{\bar{N}^{2}}-\frac{1}{\bar{N}}=R .
$$

Il est possible d'effectuer un calcul d'erreur rigoureux sur $R$ uniquement en fonction des taux de comptage mesurés et des corrections de temps mort, mais ce calcul est fastidieux. Nous donnons une formule simplifiée qui conduit au même résultat, formule établie en admettant que les valeurs moyennes et les variances des distributions mesurées $M_{n}$ et $B_{n}$ sont des quantités indépendantes et que l'incidence des corrections de temps mort est négligeable :

$$
\begin{array}{r}
\operatorname{Er}^{2}(R)=\frac{1}{\mathcal{N}(\bar{M}-\bar{B})^{2}}\left[\frac{\mu_{M}-\sigma_{M}^{4}+2\left(\mu_{B}-\sigma_{B}^{4}\right)}{(\bar{M}-\bar{B})^{2}}+\right. \\
\left.+(2 R(\bar{M}-\bar{B})-1)\left(\sigma_{M}^{2}+2 \sigma_{B}^{2}\right)\right]
\end{array}
$$

$\mu$ est le moment centré d'ordre 4 et le facteur 2, qui apparaît devant les termes de bruit, est dû au fait que l'échantillonnage du bruit dans la porte de comptage du signal est évidemment statistiquement différent de celui qui est mesuré dans la porte de comptage du bruit.

La vérification expérimentale de l'invariance théorique de $R$ en fonction de l'efficacité de détection des neutrons est un excellent critère pour tester tout 
TABLEAU I

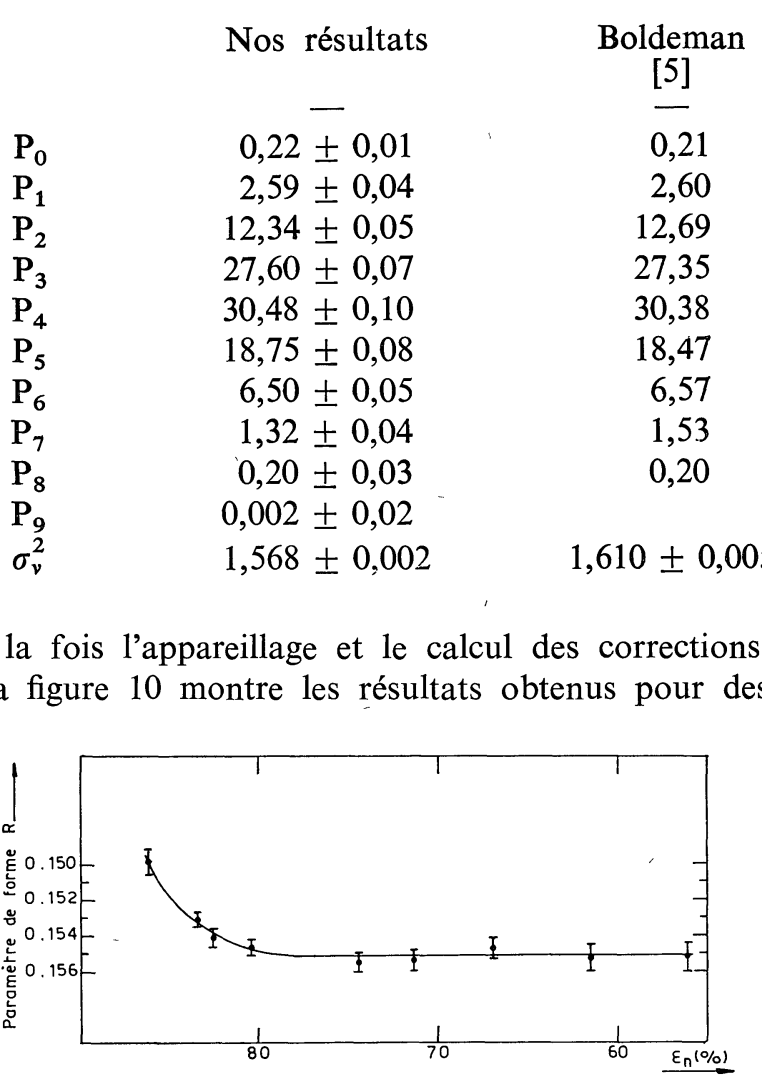

Fig. 10. - Valeur de l'invariant $R$ en fonction de l'efficacité de détection neutronique.

efficacités comprises entre $50 \%$ et $85 \%$; on constate que $R$ a bien une valeur constante tant que $\varepsilon$ reste inférieur à $82 \%$. Au-delà de cette valeur limite, il faut considérer que les mesures sont perturbées.

Le tableau I montre les résultats que nous avons obtenus concernant la fission spontanée de ${ }^{252} \mathrm{Cf}$, comparés à ceux de la littérature renormalisés pour obtenir une valeur de $v$ égale à 3,756 .

Conclusion. - Dans ce travail, nous nous proposions d'étudier un détecteur de neutrons de forte efficacité et de rechercher, afin de les prévenir, les

Soleilhac
$[10]$
-
0,24
2,61
12,66
27,42
30,32
18,36
6,67
1,51
0,23

1,625

$\begin{array}{rr}\begin{array}{c}\text { Diven } \\ {[14]}\end{array} & \begin{array}{c}\text { Hicks } \\ {[8]}\end{array} \\ - & -53 \\ 1,28 & 0,14 \\ 15,10 & 2,47 \\ 27,46 & 12,14 \\ 33,87 & 27,89 \\ 16,12 & 31,83 \\ 6,29 & 17,02 \\ 1,88 & 7,06 \\ 0,47 & 1,18 \\ & 0,26 \\ 1,639 & \\ & 1,565\end{array}$

erreurs systématiques qui pouvaient fausser les mesures.

Les principales difficultés d'ordre expérimental que nous avons rencontrées se placent au niveau des post-impulsions produites dans les tubes photomultiplicateurs. Nous n'avons jamais pu obtenir de résultats corrects, quel que soit le groupement des tubes, tant que la haute tension qui leur était appliquée était élevée. La solution que nous préconisons, de sous-polariser fortement les tubes nous a permis de nous affranchir complètement de ce défaut. Dans ces conditions, le montage électronique peut être très simplifié puisqu'une simple addition analogique des signaux de tous les P. M. est suffisante.

La constance de l'invariant de la distribution du nombre de neutrons comptés en fonction de l'efficacité de détection est le bon critère pour confirmer la validité de la mesure et l'exactitude des différentes corrections.

Comme première application de cette installation expérimentale, nous avons mesuré la variance $\sigma_{v}^{2}$ de la distribution $P_{v}$ des neutrons prompts de ${ }^{252} \mathrm{Cf}$ : avec une valeur de référence de $\bar{v}$ égale à 3,756 , nous avons trouvé $\sigma_{v}^{2}=1,568 \pm 0,002$, valeur notablement plus faible que celles publiées dans la littérature [13].

\section{Bibliographie}

[1] Poitou (J.), Nifenecker (H.) et Signarbieux (C.), Note CEA N-1282, 1970.

[2] Veyssière (A.), Beil (H.), Bergère (R.), Carlos (P.) et Leprêtre (A.), Nucl. Phys., 1970, A 159, 561.

[3] Clark (G. E.), Morrison (R. C.), O'Laughlin (J. W.) et BURKHOLdeR (H. R.), Nucl. Instr. and Methods, 1970, 84, 67.

[4] Pr. SchneIder, Université de Giessen, communication privée.

[5] Boldeman (J. W.) et Dalton (A. W.), AAEC/E, $172,1967$.

[6] Asplund-Nilsson (I.), Conde (H.) et Starfelt (N.), Nucl. Sci. and Engineering, 1963, 15, 213.

[7] Mather (D. S.), Fieldhouse (P.) et MoAT (A.), Phys. Rev., 1964, 133, B 1403.
[8] Hicks (D. A.), Isé (J.) et Pyle (R. V.), Phys. Rev., 1956, 101, 1016.

[9] Hopkins (J. C.) et Diven (B. C.), Nucl. Phys., 1963, 48, 433.

[10] Soleilhac (M.), Frehaut (J.) et Gauriau (J.), $J$. of Nucl. Energy, 1969, 23, 257.

[11] Colvin (C. W.), Conf. Proc. Helsinki, II (I. A. E. A., Vienna, 1970).

[12] NifeneCKer (H.), Nucl. Instr. and Methods, 1970, $81,45$.

[13] Signarbieux (C.), Ribrag (M.), Poitou (J.) et MATUSZEK (J.), Nucl. Instr. and Methods, 1971, 95, 585.

[14] Diven (B. C.), Martin (H. C.), Taschek (R. F.) et Terrell (J.), Phys. Rev., 1956, 101, 1012. 